\title{
A Silent Shunt on Transesophageal Echocardiography
}

\author{
Titus A*, Akkad I, Aksoy E, Ray B and Daggubati R \\ Department of Cardiology, NYU Langone Long Island School of Medicine, Winthrop Hospital, New York, USA
}

"Correspondence: Anoop Titus, Department of Cardiology, NYU Langone Long Island School of Medicine, Winthrop Hospital, New York, USA

Received on 12 February 2020; Accepted on 21 March 2020; Published on 27 April 2020

Copyright (C) 2020 Titus A, et al. This is an open access article and is distributed under the Creative Commons Attribution License, which permits unrestricted use, distribution, and reproduction in any medium, provided the original work is properly cited.

Keywords: hypertension, palpitations, dyspnea, echocardiogram, echocardiography

Abbreviations: AF: atrial fibrillation; RV: right ventricular; TEE: transesophageal echocardiography; ASD: atrial septal defect; HF: heart failure; PHTN: pulmonary hypertension

\section{Clinical Image}

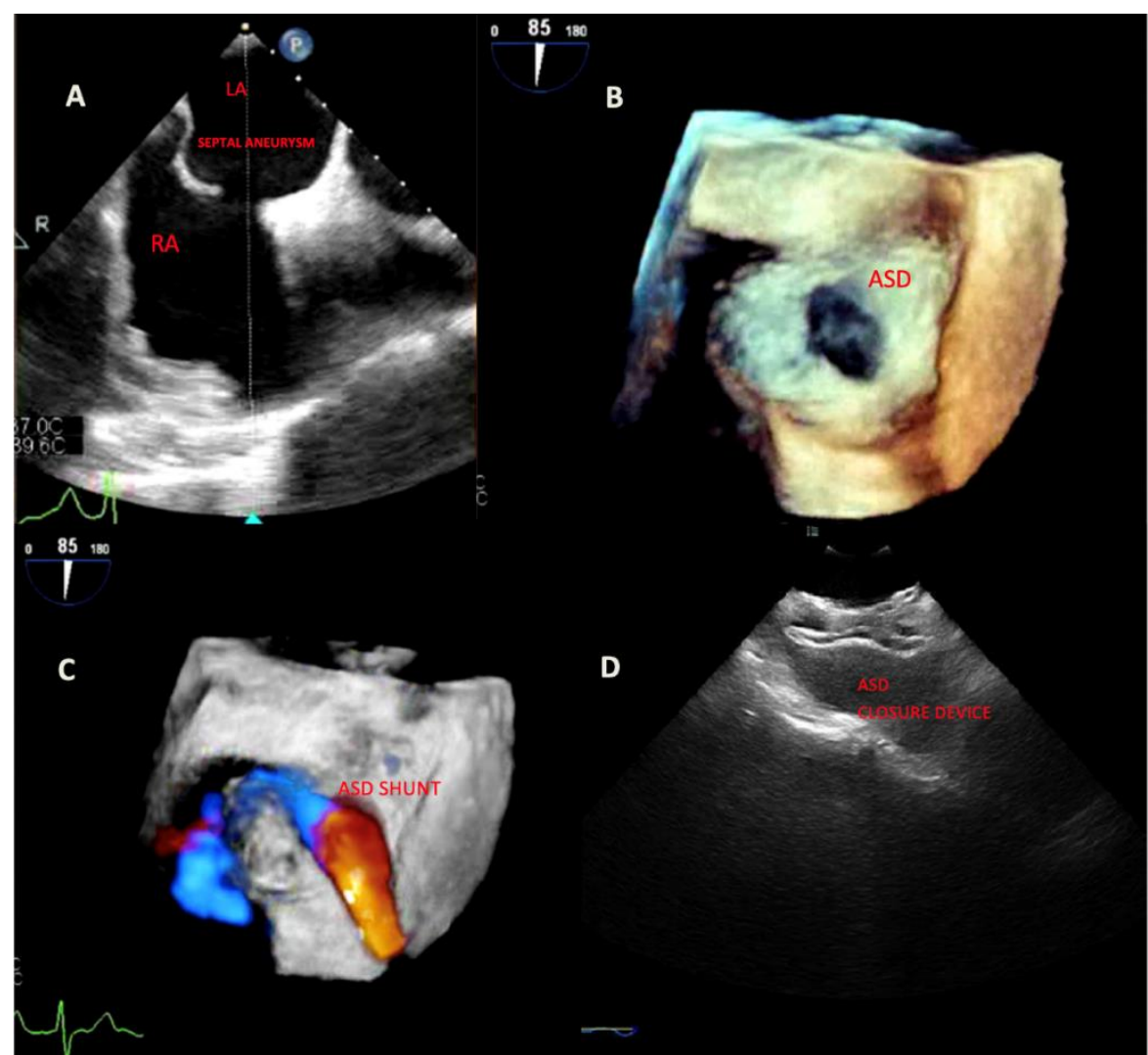

Figure 1: A) Transesophageal echocardiography showing atrial septal defect and septal aneurysm. B) 3D-echocardiography showing ASD. C) Doppler flow across the ASD. D) ASD closure device post-procedurally. 
A 69-year old male with a medical history of hypertension presented to the emergency room with complaints of palpitations, light-headedness, dyspnea and abdominal distension. On arrival he was hypotensive with irregularly irregular pulse and electrocardiogram showing atrial fibrillation (AF) with rapid ventricular response, a right axis deviation with $\mathrm{rSr}$ ' in $\mathrm{V} 1$ lead. Chest $\mathrm{X}$-ray revealed increased pulmonary congestion and pulmonary edema. Brain natriuretic peptide was 640 , consistent with elevated right sided filling pressures. Transthoracic echocardiogram revealed a preserved left ventricular ejection fraction, atrial septal aneurysm, severe right ventricular (RV) enlargement, mildly reduced RV systolic function and an unaccounted severe RV dilatation. Patient underwent transesophageal echocardiography (TEE) for left atrial appendage clot assessment and for direct current cardioversion as rhythm control strategy for AF. He was found to have a large secundum type atrial septal defect (ASD) sized 1.3 $\mathrm{cm} \times 2.2 \mathrm{~cm}$ with significant color flow Doppler and agitated saline across the defect.

ASD is believed to have an incidence of about 100/100,000 live births and represents about 1/3 of congenital heart defects. Septum secundum ASD is the most common, occurring in about $75 \%$ of all cases [1]. Usually, $65-75 \%$ cases occur in females [2]. Symptoms usually present from the fourth decade according to the defect size and shunt severity. Exercise intolerance is usually the presenting symptom. Atrial arrhythmias are seen more in patients $>40$ years of age [3]. Other features include tricuspid regurgitation, heart failure (HF) and pulmonary hypertension (PHTN).

Our patient presented with HF with AF and unexplained RV dilation. Once the ASD measurements were noted, color flow and agitated saline were utilized for evaluation of shunting degree and the need for intervention. Patient was evaluated for closure device to prevent progression of PHTN and RV failure. We performed a right sided heart catheterization to evaluate hemodynamics, which revealed $\mathrm{PCWP}=5, \mathrm{RA}=3, \mathrm{RV}=42 / 7, \mathrm{PA}=44 / 19 \mathrm{mmHg}$.

In conclusion, we believe that early identification, evaluation and management for secundum type ASD is crucial to prevent progression of shunting, RV failure and PHTN. As demonstrated, TEE is highly sensitive for diagnosis [4]. We followed the patient post-procedurally and he referred significant improvement in symptoms consistent with our hypothesis that his AF, HF was most likely related to this uncommon condition. This case highlights the importance on keeping this diagnosis in mind for proper identification and early therapeutic intervention before irreversible structural heart disease ensues.

\section{Conflicts of Interest}

Isaac Akkad, Anoop Titus, Elif Aksoy, Beevash Ray, and Ramesh Daggubati declare that they have no conflicts of interest.

\section{Human Rights Statements and Informed Consent}

All procedures followed were in accordance with the ethical standards of the responsible committee on human experimentation (institutional and national) and with the Helsinki Declaration of 1964 and later revisions. Informed consent was obtained from all patients for being included in the study.

\section{References}

1. Lin JP, Aboulhosn JA, Child JS. Hurst's The Heart. 14th ed. New York. McGraw-Hill Education. 2017. Chapter 56: Congenital heart disease in adolescents and adults; p. 1363-96.

2. Webb G, Gatzoulis MA. Atrial septal defects in the adult: recent progress and overview. Circulation. 2006;114(15):1645-53.

3. Gatzoulis MA, Freeman MA, Siu SC, et al. Atrial arrhythmia after surgical closure of atrial septal defects in adults. N Engl J Med. 1999;340(11):839-46.

4. Hausmann D, Daniel WG, Mugge A, et al. Value of transesophageal color doppler echocardiography for detection of different types of atrial septal defect in adults. J Am Soc Echocardiogr. 1992;5(5):481-88. 